\title{
Effect of Health Promotion Program on Compliance of Patients with Hypertension toward Therapeutic Regimen
}

\author{
Doaa Mohammed Sabry Abd Allah Goda ${ }^{1}$, Nadia Mohamed Taha ${ }^{2}$, \\ Abd El Fatah Hassan Frere ${ }^{3}$ and Elham Hamed Mohamed ${ }^{4}$ \\ ${ }^{I}$ B.Sc. Nursing, Faculty of Nursing, Zagazig University, Egypt \\ ${ }^{2}$ Medical Surgical Nursing, Faculty of Nursing, Zagazig University , Egypt. \\ ${ }^{3}$ Blood Vessels and Heart, Faculty of Medicine, Zagazig University, Egypt. \\ ${ }^{4}$ Medical Surgical Nursing, Faculty of Nursing, Zagazig University,Egypt.
}

Corresponding Author

Doaa Mohammed Sabry Abd-Allah

Goda

Mobile: 01092905364

Email:
doaagooda99@yahoo.
com

Key words: Hypertension, Compliance, Health Promotion Program
Background and study aim: Hypertension is a global health issue among the adult population. Compliance with therapeutic regimen is an effective step for better control of blood pressure and preventing the risk of complications. The aim of study was to evaluate the effect of health promotion program on compliance with therapeutic regimen for patients with hypertension.

Subjects and Methods: A quasi experimental design was used in this study. The study was conducted in outpatient clinic, department of cardiology. A purposive sample of 80 adult patients suffering from hypertension divided into two groups study and control were included. The study lasted from the beginning of May 2016 to the end of May 2017. Four tools were used for collection of data. A structured interview questionnaire tool for patient consisted of personal characteristics of patients and patient's knowledge regarding hypertension, Compliance assessment scale, Observation checklist and Factors affecting patient's compliance.
Results: The study finding revealed that, there was a significant improvement in knowledge, practice and compliance of patients with hypertension in study group in post and follow up program phase $\mathrm{P}=0.00$. Also, the patients showed high improved level regarding lifestyle habits. In addition to there was significant decrease in systolic, diastolic blood pressure for patients in study group.

Conclusion: It can be concluded that application of health promotion program for adult patients with hypertension showed an improvement in patients' knowledge which can reflect an improvement on their practice and compliance. It is recommended to increase awareness of patients about risk factors of hypertension to empower and motivate them to adopt healthy lifestyle to prevent the disease. This can be achieved through mass media and health education programs about the disease process and the importance of adopting healthy lifestyle.

\section{INTRODUCTION}

Hypertension is remains a major global public health challenges that has been identified as the leading risk factor for cardiovascular morbidity and mortality. Hypertension is defined as a systolic blood pressure equal to or above 140 $\mathrm{mmHg}$ and/or diastolic blood pressure equal to or above $90 \mathrm{mmHg}$. Normal levels of both systolic and diastolic blood pressure are particularly important for the effective function of vital organs such as heart, brain and kidneys as well as for overall health and well-being [1]. The prevalence of hypertension and burden of disease caused by high blood pressure (BP) is increasing in the world [2]. The World Health Organization [3] estimates that Onequarter of the world's adult population has hypertension, affects more than $26 \%$, approximately one billion of the adult people all over the world and this is likely to increase to $29 \%$ to a total of 1.56 billion by 2025 will have hypertension. In Eastern Mediterranean Region, the prevalence of hypertension was $29 \%$ and it was affected approximately 125 million individuals [4]. Egyptian National Hypertension Project showed that hypertension is common among Egyptians. The 
prevalence of hypertension is $26.3 \%$. The problem is complicated by the low awareness rates, only $38 \%$ of hypertensive Egyptians aware of having high blood pressure [5].

Compliance with therapeutic regimen is considered the main theme in the management of the patient with hypertension. Compliance is defined as the extent to which a patient's behaviour (taking medication, following a diet, modifying habits or attending clinics) coincides with healthcare giver advice [6]. Uncontrolled hypertension is caused by non-compliance to the therapeutic regimen. For patients with hypertension, compliance depending on compliance with dietary management, drug regimen, Lifestyle habites and clinic visits every 1-3 months. patient's knowledge, patients' beliefs and motivation towards the therapy help the patients to improve their compliance, thus will prevent the complications of hypertension which are debilitating and if not prevented can increase the burden of a disease that is already on the increase [7].

Health promotion is defined as the "science and art of helping people change their lifestyle to move toward a state of optimal health, which is a balance of physical, emotional, social, spiritual, and intellectual health. There are different theories and models that are used to explain health promotion and incorporate it into practice. The most common model is the Health Belief Model [8]. Health Belief Model is concerned with what people perceive, or believe, to be true about themselves in relation to their health. The HBM was also widely used to explain a range of health behavior. There are six constructs to the HBM: perceived susceptibility, perceived severity, perceived benefits, perceived barriers, self-efficacy, and cues to action. The way persons relate themselves to each of these areas is predictive of how likely they are to engage or not engage in a certain behavior. In hypertension the HBM bases on studying compliances with lifestyle modification and antihypertensive medication [9].

The aim of the study was to evaluate the effect of health promotion program on compliance with therapeutic regimen for patients with hypertension.

\section{SUBJECTS AND METHODS}

A quasi experimental design was utilized in the study. Study was conducted in outpatient clinics at Zagazig University Hospitals. Field work of this study was executed in 12 months, starting from May 2016 to May 2017.

\section{Subjects:}

A purposive sample of 80 adult patients with hypertension. They were divided randomly into two groups "study" and "control" (40 patients for each group).

Tools for data collection: Four tools were used for data collection:

Tool I: A Structured interview questionnaire tool for patients was designed by the researcher after revising of related literature and opinions of expertise for content of validity and included the following four parts:

- Part 1: Demographic characteristics of patients e.g. (age, sex, marital status, occupation, level of education ....etc)

- Part 2: Medical history of patients e.g. (chronic illness, family history, and disease duration)

- Part 3: Life style habites of patients (Pre/ Post/follow up test). It included Nutritional habits, Physical activity, Weight control .etc).

- Part 4: Patient's knowledge regarding hypertension (Pre/ Post / follow up test). It included meaning of a blood pressure reading, normal value of $\mathrm{Bp}$ for adults, hypertension is chronic disease, primary hypertension is the most common type ....etc.

Tool II: The Compliance assessment scale (Pre/ Post/ follow up test): It was used to assess the compliance of patients with hypertension toward therapeutic regimen [10].

Tool III: Observation checklist (Pre/ Post/ follow up test): A structured observational checklist was developed by the researcher to evaluate patients ' practice It included: BP measurement, Breathing exercises, Progressive Muscle Relaxation, Meditation and Guided Imagery as guided by Lynn \& LeBon [11], Sue, DeLaune \& Ladner [12], Dave \& Makwana [13] and Madhava \& Deepa [14].

Tool IV: Factors affecting patient's compliance (Pre, Post and follow up test): a Structure Interview Questionnaire sheet, It was designed by the researcher based on Health Belief model, to measure factors affecting compliance with antihypertensive therapy. It included six constructs: Perceived severity, Perceived susceptibility, Perceived benefits, Perceived barriers, Self- Efficacy and Cues to Action.

\section{Health promotion program:}

It was designed by the researcher after revising of related literature. It consisted of theoretical and 
practical parts. Theoretical part implemented through five sessions. Each session took 30 minutes. It included definition of hypertension, classification, manifestations, risk factors, complications, and treatment.....etc. Practical part implemented through nine sessions. Each session took 30- 45 minutes. It included Blood pressure measurement, Breathing Exercises and Progressive Muscle Relaxation exercises ....etc. Patients were grouped; each group included 4-5 patients.

\section{Content validity and Reliability:}

Content validity was used for the modified tools and the designed booklet to determine whether the tools covered the aim or not. It developed by a jury of 5 experts four professors from faculty of Nursing, Zagazig University and one lecturer of cardiology from the Faculty of Medicine, Zagazig University. Reliability was done by using Cronbach test and retest [15]. It was used to examine whether the observation checklist, compliance assessment scale had internal consistency or not. The test was done and the agreement percentage was $97 \%$.

The implementation phase for data collection started as following: The selection of the patients, the collection of data, and the implementation of the Health promotion program lasted over a period of 12months, starting from May 2016 to May 2017, Two months for pre- test (from beginning May, 2016 to the end of June, 2016), Six months implementation the program and posttest (from beginning of July 2016 to end of December 2016), 3 months after posttest follow up test was done which lasted 2 months (from beginning of April to end of May 2017). The questionnaire sheet was designed by the researcher. Data used was collected four day from the outpatient clinics of cardiology, from 9:00 am to 1:00 pm where the program was implemented. Patients were grouped; each group included 4-5 patients. It was necessary for the researcher to introduce herself for the patients and explain the purpose of the study. The data was collected in a simplified Arabic language. The study program consisted of 16 sessions; one session of them to identify the objective and the importance of the program. One third of the sessions (5) were theoretical, and two thirds (9) were practical and one session for revision.

\section{Administrative and Ethical considerations:}

The study was ethically approved from the dean of the faculty of Nursing, the manager of Zagazig University Hospitals, the head of outpatient clinics of cardiology, ethics committee at the faculty of nursing and from Ethical committee of faculty of medicine.

\section{Statistical Design:}

All collected data were organized, categorized, tabulated, entered, and analyzed by using SPSS (Statistical Package for Social Sciences); a software program version 14 , which was applied to frequency tables and statistical significance. The statistical significance and associations were assessed using, the arithmetic mean, the standard deviation (SD), Wilcoxon Signed Ranks test ( $Z$ test), Paired sample t test (T test), Pearson chisquare test (X2) and Pearson Correlation (r) to detect the relation between the variables.

\section{RESULTS}

The first part of our results was the Demographic characteristics and medical history for patients with hypertension in both study and control groups including; gender, age, residence, marital Status, education, occupation and income (Table 1).

The second part of our results was concerned with the Patients' behaviour toward Lifestyle habites in both patients groups throughout the study phases, It clarified that Mean scores for total and all dimensions of lifestyle habites such as nutritional habites, physical activity, weight control and stress management were increased (improved) significantly between pre / post and pre / follow up program phase in the study group $\mathrm{P}=0.00$ compared to the smoking habites there was no improvement for patients in both study and control group throughout the study and follow up phases (Table 2).

The third part of our results was concerned with the Patients' knowledge about hypertension in both patients groups throughout the study phases. It revealed that the patients in study group had satisfactory level of knowledge about hypertension, diet, and treatment regimen between pre/post and pre/follow up program phase $(95.0 \%, 82.5 \%)$ respectively, While no significant differences occurred in the control group. The table showed statistical significant difference in study group throughout the study phases $\mathrm{P}=0.00$ (Table 3 ).

The fourth part of our results was concerned with the Patients' compliance toward therapeutic regimen obtained by patients in both groups throughout the study phase. It represented that the patients in study group were good compliant 
toward therapeutic regimen regarding diet regimen, treatment regimen and comply with lifestyle habites between pre/post and pre/follow up program phase $(80.0 \%, 70.0 \%)$ respectively While no significant differences occurred in the control group. The table showed statistical significant difference in study group throughout the study phases $\mathrm{P}=0.00$ (Table 4 ).

The fifth part of our results was concerned with the Patients' practice obtained by patients with hypertension in both groups throughout the study phase. It showed that, the patients in study group had satisfactory practice related to BP measurement, breathing exercises, Progressive Muscle Relaxation, meditation, guided Imagery between pre/post and pre/follow up program phase $\mathrm{P}=0.001$ compared to control group none of patients had satisfactory practice throughout the study phases (Table 5).

The sixth part of our results was concerned with the Factors affecting compliance with therapeutic regimen for patients based on the HBM in both patients groups throughout the study phases. It demonstrated that, positive changes in perception were observed in the study group for all HBM constructs except, cues to action between pre/ post and pre/follow up program phase $\mathrm{P}=0.001$, $0.002,0.003,0.004$. While no significant differences occurred in the control group (Table 6).

The seventh part of our results demonstrated that there was statistical significant relation between the patients' knowledge and their demographic characteristics, only among their educational level in study group in post program phase $(\mathrm{p}<0.05)$, while, there was statistical significant relation between the patients' compliance and their demographic characteristics, among their age and income in study group in post program phase (pvalue $<0.05$ ), also there was statistical significant relation between total compliance score and HBM among perceived severity, perceived benefits and Self-Efficacy in study group in post phase (p-value $<0.05$ ), there was statistically positive correlation between patients' knowledge and practice in post and follow up program phase, there was statistically positive correlation between patients' practice and compliance in post program phase. Also there was statistically positive correlation between patients' knowledge and compliance in post program phase and versus (Tables 7, 8, 9, 10). 
Table (1): Demographic Characteristics for patients in both groups $(n=80)$

\begin{tabular}{|c|c|c|c|c|c|}
\hline \multirow{3}{*}{ Demographic characteristics } & \multicolumn{4}{|c|}{ Group } & \multirow{3}{*}{ MCP } \\
\hline & \multicolumn{2}{|c|}{ Study $(n=40)$} & \multicolumn{2}{|c|}{ Control $(n=40)$} & \\
\hline & No & $\%$ & No & $\%$ & \\
\hline Age in years & \multirow{2}{*}{15} & \multirow{2}{*}{37.5} & \multirow{2}{*}{17} & \multirow{2}{*}{42.5} & \multirow{6}{*}{0.397} \\
\hline $25-50$ & & & & & \\
\hline $51-65$ & 25 & 62.5 & 23 & 57.5 & \\
\hline Range & \multicolumn{2}{|c|}{$25-65$} & \multicolumn{2}{|c|}{$25-65$} & \\
\hline Mean $\pm \mathrm{SD}$ & \multicolumn{2}{|c|}{$54.1 \pm 11.0$} & \multicolumn{2}{|c|}{$53.9 \pm 10.5$} & \\
\hline Median & \multicolumn{2}{|c|}{54.5} & \multicolumn{2}{|c|}{53.5} & \\
\hline \multicolumn{5}{|l|}{ Gender } & \multirow{3}{*}{0.483} \\
\hline Male & 10 & 25.0 & 13 & 32.5 & \\
\hline Female & 30 & 75.0 & 27 & 67.5 & \\
\hline \multicolumn{5}{|l|}{ Residence } & \multirow{3}{*}{0.776} \\
\hline urban & 8 & 20.0 & 7 & 17.5 & \\
\hline Rural & 32 & 80.0 & 33 & 82.5 & \\
\hline \multicolumn{5}{|l|}{ Marital Status } & \multirow{4}{*}{0.345} \\
\hline Married & 30 & 75.0 & 29 & 72.5 & \\
\hline Single & 8 & 20.0 & 6 & 15.0 & \\
\hline Widow & 2 & 5.0 & 5 & 12.5 & \\
\hline Education & \multirow[b]{2}{*}{26} & \multirow[b]{2}{*}{65.0} & \multirow[b]{2}{*}{30} & \multirow[b]{2}{*}{75.0} & \multirow{4}{*}{0.857} \\
\hline Illiterate & & & & & \\
\hline School education & 10 & 25.0 & 9 & 22.5 & \\
\hline University & 4 & 10.0 & 1 & 2.5 & \\
\hline Occupation & \multirow[b]{2}{*}{2} & & & & \\
\hline Work & & 5.0 & 2 & 5.0 & 0.840 \\
\hline Don't work & 38 & 95.0 & 38 & 95.0 & \\
\hline Income & & & & & \\
\hline Enough & 12 & 30.0 & 10 & 25.0 & 1.317 \\
\hline Not enough & 28 & 70.0 & 30 & 75.0 & \\
\hline Crowding index & & & & & \\
\hline$<2$ & 13 & 32.5 & 14 & 35.0 & 0.259 \\
\hline$\geq 2$ & 27 & 67.5 & 26 & 65.0 & \\
\hline
\end{tabular}


Table (2): Mean scores of Lifestyle habites for patients with hypertension in both groups $(\mathrm{n}=80)$

\begin{tabular}{|c|c|c|c|c|c|c|}
\hline \multirow{3}{*}{ Lifestyle items } & \multicolumn{3}{|c|}{ Study group $(n=40)$} & \multicolumn{3}{|c|}{ Control group $(n=40)$} \\
\hline & Pre & Post & $\begin{array}{c}\text { Follow } \\
\text { Up }\end{array}$ & Pre & Post & $\begin{array}{c}\text { Follow } \\
\text { Up }\end{array}$ \\
\hline & $\begin{array}{c}\text { Mean } \pm \\
\text { SD }\end{array}$ & $\begin{array}{c}\text { Mean } \pm \\
\text { SD }\end{array}$ & $\begin{array}{c}\text { Mean } \pm \\
\text { SD }\end{array}$ & $\begin{array}{c}\text { Mean } \pm \\
\text { SD }\end{array}$ & $\begin{array}{c}\text { Mean } \pm \\
\text { SD }\end{array}$ & $\begin{array}{c}\text { Mean } \pm \\
\text { SD }\end{array}$ \\
\hline Nutritional habites & $2.17 \pm 0.27$ & $2.61 \pm 0.23$ & $2.58 \pm 0.22$ & $2.27 \pm 0.35$ & $2.33 \pm 0.31$ & $2.31 \pm 0.35$ \\
\hline T-test (P1) & \multicolumn{3}{|c|}{$17.059(0.000)^{*}$} & \multicolumn{3}{|c|}{$3.240(0.072)$} \\
\hline T-test (P2) & \multicolumn{3}{|c|}{$15.715(0.000)^{*}$} & \multicolumn{3}{|c|}{$2.202(0.083)$} \\
\hline Physical activity & $1.68 \pm 0.46$ & $2.21 \pm 0.42$ & $2.24 \pm 0.36$ & $1.73 \pm 0.46$ & $1.77 \pm 0.49$ & $1.76 \pm 0.49$ \\
\hline T-test (P1) & \multicolumn{3}{|c|}{$12.611(0.000)^{*}$} & \multicolumn{3}{|c|}{$2.449(0.069)$} \\
\hline T-test (P2) & \multicolumn{3}{|c|}{$12.969(0.000)^{*}$} & \multicolumn{3}{|c|}{$2.138(0.078)$} \\
\hline weight control & $2.76 \pm 0.31$ & $2.94 \pm 0.24$ & $2.91 \pm 0.25$ & $2.81 \pm 0.23$ & $2.83 \pm 0.22$ & $2.80 \pm 0.24$ \\
\hline T-test (P1) & \multicolumn{3}{|c|}{$6.773(0.000)^{*}$} & \multicolumn{3}{|c|}{$1.704(0.096)$} \\
\hline T-test (P2) & \multicolumn{3}{|c|}{$5.877(0.000)^{*}$} & \multicolumn{3}{|c|}{$0.650(0.519)$} \\
\hline Smoking habites & $2.77 \pm 0.22$ & $2.78 \pm 0.21$ & $2.79 \pm 0.22$ & $2.05 \pm 0.3$ & $2.02 \pm 0.34$ & $2.02 \pm 0.34$ \\
\hline T-test (P1) & \multicolumn{3}{|c|}{$2.077(0.107)$} & \multicolumn{3}{|c|}{$1.262(0.214)$} \\
\hline T-test (P2) & \multicolumn{3}{|c|}{$2.254(0.081)$} & \multicolumn{3}{|c|}{$1.320(0.118)$} \\
\hline Stress management & $2.12 \pm 0.35$ & $2.47 \pm 0.24$ & $2.50 \pm 0.21$ & $2.22 \pm 0.30$ & $2.26 \pm .29$ & $2.25 \pm 0.29$ \\
\hline T-test (P1) & \multicolumn{3}{|c|}{$11.343(0.000)^{*}$} & \multicolumn{3}{|c|}{$3.812(0.063)$} \\
\hline T-test (P2) & \multicolumn{3}{|c|}{$11.720(0.000)^{*}$} & \multicolumn{3}{|c|}{$3.716(0.071)$} \\
\hline Total lifestyle score & $11.45 \pm 1.5$ & $12.75 \pm 1.24$ & $12.64 \pm 1.1$ & $11.84 \pm 1.5$ & $12.01 \pm 1.6$ & $11.97 \pm 1.5$ \\
\hline T-test (P1) & \multicolumn{3}{|c|}{$24.633(0.000)^{*}$} & \multicolumn{3}{|c|}{$5.050(0.933)$} \\
\hline T-test (P2) & \multicolumn{3}{|c|}{$19.875(0.000)^{*}$} & \multicolumn{3}{|c|}{$3.374(0.882)$} \\
\hline
\end{tabular}

$* \mathrm{P}<0.05$ (significant) $\quad \mathrm{P} 1$ : Pre/post $\quad \mathrm{P} 2:$ Pre/ FU

Table (3): Patients' knowledge in both groups throughout the study phases $(n=80)$

\begin{tabular}{|c|c|c|c|c|c|c|c|c|c|c|c|c|}
\hline \multirow{4}{*}{ Knowledge } & \multicolumn{12}{|c|}{ Group } \\
\hline & \multicolumn{6}{|c|}{ Study $(n=40)$} & \multicolumn{6}{|c|}{ Control $(n=40)$} \\
\hline & \multicolumn{2}{|c|}{ Pre } & \multicolumn{2}{|c|}{ Post } & \multicolumn{2}{|c|}{ Follow Up } & \multicolumn{2}{|c|}{ Pre } & \multicolumn{2}{|c|}{ Post } & \multicolumn{2}{|c|}{$\begin{array}{c}\text { Follow } \\
\text { Up }\end{array}$} \\
\hline & No & $\%$ & No & $\%$ & No & $\%$ & No & $\%$ & No & $\%$ & No & $\%$ \\
\hline \multicolumn{13}{|c|}{ knowledge about disease } \\
\hline Satisfactory & 2 & 5.0 & 39 & 97.5 & 31 & 77.5 & 2 & 5.0 & 2 & 5.0 & 2 & 5.0 \\
\hline Z-test (P1) & \multicolumn{6}{|c|}{$6.083(.000 *)$} & \multicolumn{6}{|c|}{$.000(1.000)$} \\
\hline Z-test (P2) & \multicolumn{6}{|c|}{$5.745(.000 *)$} & \multicolumn{6}{|c|}{$.000(1.000)$} \\
\hline \multicolumn{13}{|c|}{ knowledge about diet regimen } \\
\hline Satisfactory & 3 & 7.5 .0 & 37 & 92.5 & 33 & 82.5 & 5 & 12.5 & 5 & 12.5 & 5 & 12.5 \\
\hline Z -test (P1) & \multicolumn{6}{|c|}{$5.831(.000 *)$} & \multicolumn{6}{|c|}{$.000(1.000)$} \\
\hline Z -test (P2) & \multicolumn{6}{|c|}{$5.568(.000 *)$} & \multicolumn{6}{|c|}{$.000(1.000)$} \\
\hline \multicolumn{13}{|c|}{ knowledge about treatment regimen } \\
\hline Satisfactory & 3 & 7.5 & 38 & 95.0 & 36 & 90.0 & 3 & 7.5 & 3 & 7.5 & 3 & 7.5 \\
\hline Z -test (P1) & \multicolumn{6}{|c|}{$5.916(.000 *)$} & \multicolumn{6}{|c|}{$.000(1.000)$} \\
\hline Z -test (P2) & \multicolumn{6}{|c|}{$5.578(.000 *)$} & \multicolumn{6}{|c|}{$.000(1.000)$} \\
\hline \multicolumn{13}{|l|}{ Total knowledge } \\
\hline Satisfactory & 3 & 7.5 & 38 & 95.0 & 33 & 82.5 & 4 & 10.0 & 4 & 10.0 & 4 & 10.0 \\
\hline Z -test (P1) & \multicolumn{6}{|c|}{$5.604(.000 *)$} & \multicolumn{6}{|c|}{$.000(1.000)$} \\
\hline Z -test (P2) & \multicolumn{6}{|c|}{$5.477(.000 *)$} & \multicolumn{6}{|c|}{$.000(1.000)$} \\
\hline
\end{tabular}

$* \mathrm{P}<0.05$ (significant) P1: Pre/post $\quad$ P2: Pre/ FU


Table (4): Patients' compliance score in both groups throughout the study phases $(\mathrm{n}=80)$

\begin{tabular}{|c|c|c|c|c|c|c|c|c|c|c|c|c|}
\hline \multirow{4}{*}{ Compliance } & \multicolumn{12}{|c|}{ Group } \\
\hline & \multicolumn{6}{|c|}{ Study $(n=40)$} & \multicolumn{6}{|c|}{ Control $(n=40)$} \\
\hline & \multicolumn{2}{|c|}{ Pre } & \multicolumn{2}{|c|}{ Post } & \multicolumn{2}{|c|}{ Follow Up } & \multicolumn{2}{|c|}{ Pre } & \multicolumn{2}{|c|}{ Post } & \multicolumn{2}{|c|}{ Follow Up } \\
\hline & No & $\%$ & No & $\%$ & No & $\%$ & No & $\%$ & No & $\%$ & No & $\%$ \\
\hline \multicolumn{13}{|c|}{ Compliance with diet regimen } \\
\hline Good compliance & 3 & 7.5 & 28 & 70.0 & 24 & 60.0 & 3 & 7.5 & 3 & 7.5 & 3 & 7.5 \\
\hline $\mathrm{Z}$-test (P1) & \multicolumn{6}{|c|}{$4.600(.000 *)$} & \multicolumn{6}{|c|}{$.000(1.000)$} \\
\hline Z -test (P2) & \multicolumn{6}{|c|}{$2.985(.003 *)$} & \multicolumn{6}{|c|}{$.000(1.000)$} \\
\hline \multicolumn{13}{|c|}{ Compliance with treatment regimen } \\
\hline Good compliance & 5 & 12.5 & 37 & 92.5 & 33 & 82.5 & 8 & 20.0 & 8 & 20.0 & 8 & 20.0 \\
\hline Z -test (P1) & \multicolumn{6}{|c|}{$5.048(.000 *)$} & \multicolumn{6}{|c|}{$.000(1.000)$} \\
\hline Z -test (P2) & \multicolumn{6}{|c|}{$4.600(.000 *)$} & \multicolumn{6}{|c|}{$.000(1.000)$} \\
\hline \multicolumn{13}{|c|}{ Compliance with Lifestyle habites } \\
\hline Good compliance & 2 & 5.0 & 34 & 85.0 & 30 & 75.0 & 1 & 2.5 & 1 & 2.5 & 1 & 2.5 \\
\hline Z -test (P1) & \multicolumn{6}{|c|}{$5.916(.000 *)$} & \multicolumn{6}{|c|}{$.000(1.000)$} \\
\hline Z -test (P2) & \multicolumn{6}{|c|}{$5.568(.000 *)$} & \multicolumn{6}{|c|}{$.000(1.000)$} \\
\hline \multicolumn{13}{|l|}{ Total compliance } \\
\hline Good compliance & 3 & 7.5 & 32 & 80.0 & 28 & 70.0 & 4 & 10.0 & 4 & 10.0 & 4 & 10.0 \\
\hline Z -test (P1) & \multicolumn{6}{|c|}{$5.578(.000 *)$} & \multicolumn{6}{|c|}{$.000(1.000)$} \\
\hline $\mathrm{Z}$-test (P2) & \multicolumn{6}{|c|}{$5.385(.000 *)$} & \multicolumn{6}{|c|}{$.000(1.000)$} \\
\hline
\end{tabular}

$* \mathrm{P}<0.05$ (significant) $\mathrm{P} 1$ : Pre/post $\quad \mathrm{P} 2:$ Pre/ FU

Table (5): Patients' practices obtained by patients with hypertension in both groups throughout the study phases $(n=80)$

\begin{tabular}{|c|c|c|c|c|c|c|c|c|c|c|c|c|}
\hline \multirow{4}{*}{ Patients' practices } & \multicolumn{12}{|c|}{ Group } \\
\hline & \multicolumn{6}{|c|}{ Study $(n=40)$} & \multicolumn{6}{|c|}{ Control $(n=40)$} \\
\hline & \multicolumn{2}{|c|}{ Pre } & \multicolumn{2}{|c|}{ Post } & \multicolumn{2}{|c|}{ Follow Up } & \multicolumn{2}{|c|}{ Pre } & \multicolumn{2}{|c|}{ Post } & \multicolumn{2}{|c|}{$\overline{\text { Follow Up }}$} \\
\hline & No & $\%$ & No & $\%$ & No & $\%$ & No & $\%$ & No & $\%$ & No & $\%$ \\
\hline \multicolumn{13}{|l|}{ BP measurement } \\
\hline Satisfactory & 3 & 7.5 & 36 & 90.0 & 35 & 87.5 & 0 & 0.0 & 0 & 0.0 & 0 & 0.0 \\
\hline$\overline{\mathbf{P}(\mathbf{P 1})}$ & \multicolumn{6}{|c|}{$0.001 *$} & \multicolumn{6}{|c|}{-} \\
\hline$\overline{\mathbf{P}(\mathbf{P} 2)}$ & \multicolumn{6}{|c|}{$0.001^{*}$} & \multicolumn{6}{|c|}{-} \\
\hline \multicolumn{13}{|l|}{ Breathing exercises } \\
\hline Satisfactory & 2 & 5.0 & 40 & 100.0 & 39 & 97.5 & 0 & 0.0 & 0 & 0.0 & 0 & 0.0 \\
\hline $\mathbf{P}(\mathbf{P 1})$ & \multicolumn{6}{|c|}{$0.001^{*}$} & \multicolumn{6}{|c|}{-} \\
\hline $\mathbf{P}(\mathbf{P} 2)$ & \multicolumn{6}{|c|}{$0.001^{*}$} & \multicolumn{6}{|c|}{-} \\
\hline \multicolumn{13}{|c|}{ Progressive Muscle Relaxation } \\
\hline Satisfactory & 0 & 0.0 & 40 & 100.0 & 36 & 90.0 & 0 & 0.0 & 0 & 0.0 & 0 & 0.0 \\
\hline$\overline{\mathbf{P}(\mathbf{P} 1)}$ & \multicolumn{6}{|c|}{$0.001 *$} & \multicolumn{6}{|c|}{-} \\
\hline$\overline{\mathbf{P}(\mathbf{P} 2)}$ & \multicolumn{6}{|c|}{$0.001^{*}$} & \multicolumn{6}{|c|}{-} \\
\hline \multicolumn{13}{|l|}{ Meditation } \\
\hline Satisfactory & 0 & 0.0 & 39 & 97.5 & 32 & 80.0 & 0 & 0.0 & 0 & 0.0 & 0 & 0.0 \\
\hline $\mathbf{P}(\mathbf{P} 1)$ & \multicolumn{6}{|c|}{$0.001 *$} & \multirow{2}{*}{\multicolumn{6}{|c|}{-}} \\
\hline $\mathbf{P}(\mathbf{P} 2)$ & \multicolumn{6}{|c|}{$0.001^{*}$} & & \multicolumn{5}{|c|}{-} \\
\hline $\begin{array}{l}\text { Guided Imagery } \\
\text { Satisfactory }\end{array}$ & 2 & 5.0 & 40 & 100.0 & 39 & 97.5 & 0 & 0.0 & 0 & 0.0 & 0 & 0.0 \\
\hline$\overline{P(\mathbf{P} 1)}$ & & & & $001 *$ & & & & & & 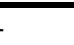 & & \\
\hline$\overline{\mathbf{P}(\mathbf{P} 2)}$ & & & & 001* & & & & & & 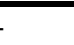 & & \\
\hline $\begin{array}{c}\text { Total practice } \\
\text { Satisfactory } \\
\end{array}$ & 2 & 5.0 & 38 & 95.0 & 34 & 85.00 & 0 & 0.0 & 0 & 0.0 & 0 & 0.0 \\
\hline$\overline{P(\mathbf{P} 1)}$ & & & & 001* & & & & & & & & \\
\hline $\bar{P}(\mathbf{P 2})$ & & & & 001* & & & & & & & & \\
\hline
\end{tabular}

\begin{tabular}{llll}
\hline P: Friedman test for repeated measures & $* \mathrm{P}<0.05$ (significant) & $\mathrm{P} 1$ : Pre/post & $\mathrm{P} 2:$ Pre/ FU
\end{tabular} 
Table (6): Perception level pertaining of patients with hypertension in both groups throughout the study phases $(\mathrm{HBM})(\mathrm{n}=80)$

\begin{tabular}{|c|c|c|c|c|c|c|}
\hline \multirow{3}{*}{$\begin{array}{c}\text { Health Belief Model } \\
\text { items }\end{array}$} & \multicolumn{3}{|c|}{ Study $(n=40)$} & \multicolumn{3}{|c|}{ Control $(n=40)$} \\
\hline & \multicolumn{3}{|c|}{ Mean \pm SD } & \multicolumn{3}{|c|}{ Mean \pm SD } \\
\hline & Pre & Post & Follow Up & Pre & Post & Follow Up \\
\hline Perceived severity & $11.5 \pm 1.3$ & $16.9 \pm 0.9$ & $16.9 \pm 0.9$ & $12.1 \pm 2.1$ & $11.9 \pm 1.8$ & $11.9 \pm 1.8$ \\
\hline $\mathbf{P}+(\mathbf{P 1})$ & \multicolumn{3}{|c|}{$0.001 *$} & \multicolumn{3}{|c|}{0.328} \\
\hline $\mathbf{P}+(\mathbf{P} 2)$ & \multicolumn{3}{|c|}{$0.001 *$} & \multicolumn{3}{|c|}{0.328} \\
\hline Perceived susceptibility & $5.0 \pm 0.6$ & $10.4 \pm 0.9$ & $10.4 \pm 0.9$ & $6.5 \pm 0.8$ & $6.6 \pm 0.9$ & $6.5 \pm 0.8$ \\
\hline $\mathbf{P}+($ P1) & \multicolumn{3}{|c|}{$0.001 *$} & \multicolumn{3}{|c|}{0.488} \\
\hline $\mathbf{P}+(\mathbf{P 2})$ & \multicolumn{3}{|c|}{$0.001 *$} & \multicolumn{3}{|c|}{0.530} \\
\hline Perceived Benefits & $2.0 \pm 0.5$ & $11.5 \pm 0.4$ & $11.5 \pm 0.4$ & $2.2 \pm 0.5$ & $3.1 \pm 0.6$ & $3.1 \pm 0.6$ \\
\hline $\mathbf{P}+($ P1) & \multicolumn{3}{|c|}{$0.001 *$} & \multicolumn{3}{|c|}{0.851} \\
\hline $\mathbf{P}+(\mathbf{P 2})$ & \multicolumn{3}{|c|}{$0.001 *$} & \multicolumn{3}{|c|}{0.851} \\
\hline Perceived Barriers & $9.5 \pm 1.7$ & $12.7 \pm 1.5$ & $11.6 \pm 1.4$ & $7.6 \pm 1.1$ & $7.5 \pm 1.2$ & $7.5 \pm 1.2$ \\
\hline $\mathbf{P}+($ P1) & \multicolumn{3}{|c|}{$0.003 *$} & \multicolumn{3}{|c|}{0.920} \\
\hline $\mathbf{P}+($ P2) & \multicolumn{3}{|c|}{$0.002 *$} & \multicolumn{3}{|c|}{0.920} \\
\hline Self-Efficacy & $3.5 \pm 0.9$ & $5.2 \pm 1.0$ & $4.5 \pm 1.6$ & $3.7 \pm 0.8$ & $3.6 \pm 0.9$ & $3.5 \pm 1.2$ \\
\hline P+( P1) & \multicolumn{3}{|c|}{$0.004 *$} & \multicolumn{3}{|c|}{0.907} \\
\hline P+( P2) & \multicolumn{3}{|c|}{$0.002 *$} & \multicolumn{3}{|c|}{0.899} \\
\hline Cues to Action & $6.0 \pm 1.2$ & $6.3 \pm 1.1$ & $6.2 \pm 1.4$ & $6.7 \pm 1.3$ & $6.7 \pm 1.4$ & $6.7 \pm 1.4$ \\
\hline $\mathbf{P}+(\mathbf{P 1})$ & \multicolumn{3}{|c|}{0.114} & \multicolumn{3}{|c|}{0.150} \\
\hline $\mathbf{P}+(\mathbf{P 2})$ & \multicolumn{3}{|c|}{0.954} & \multicolumn{3}{|c|}{0.150} \\
\hline
\end{tabular}

$\mathrm{P}+$ : $\mathrm{P}$ value of Paired t-test $\quad * \mathrm{P}<0.05$ (significant) $\mathrm{P} 1$ : Pre/post $\quad \mathrm{P} 2$ : Pre/ FU 
Table (7): Relation between total satisfactory knowledge score about hypertension and Demographic characteristics for patients in both groups throughout the study phases

\begin{tabular}{|c|c|c|c|c|c|c|c|c|c|c|c|c|}
\hline \multirow{5}{*}{$\begin{array}{l}\text { Demographic } \\
\text { characteristics }\end{array}$} & \multicolumn{6}{|c|}{ Study } & \multicolumn{6}{|c|}{ Control } \\
\hline & \multicolumn{2}{|c|}{ Pre } & \multicolumn{2}{|c|}{ Post } & \multicolumn{2}{|c|}{ Follow Up } & \multicolumn{2}{|c|}{ Pre } & \multicolumn{2}{|c|}{ Post } & \multicolumn{2}{|c|}{ Follow Up } \\
\hline & \multicolumn{2}{|c|}{$\begin{array}{c}\text { Total } \\
\text { knowledge }\end{array}$} & \multicolumn{2}{|c|}{$\begin{array}{c}\text { Total } \\
\text { knowledge }\end{array}$} & \multicolumn{2}{|c|}{$\begin{array}{c}\text { Total } \\
\text { knowledge }\end{array}$} & \multicolumn{2}{|c|}{$\begin{array}{c}\text { Total } \\
\text { knowledge }\end{array}$} & \multicolumn{2}{|c|}{$\begin{array}{c}\text { Total } \\
\text { knowledge }\end{array}$} & \multicolumn{2}{|c|}{$\begin{array}{c}\text { Total } \\
\text { knowledge }\end{array}$} \\
\hline & \multicolumn{2}{|c|}{ Satisfactory } & \multicolumn{2}{|c|}{ Satisfactory } & \multicolumn{2}{|c|}{ Satisfactory } & \multicolumn{2}{|c|}{ Satisfactory } & \multicolumn{2}{|c|}{ Satisfactory } & \multicolumn{2}{|c|}{ Satisfactory } \\
\hline & $\mathbf{X}^{2}$ & 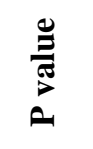 & $\mathbf{X}^{2}$ & 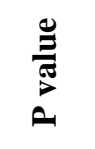 & $\mathbf{X}^{2}$ & 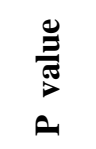 & $\mathbf{X}^{2}$ & 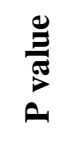 & $\mathbf{X}^{2}$ & 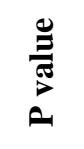 & $\mathbf{X}^{2}$ & 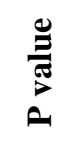 \\
\hline \multicolumn{13}{|l|}{ Age in years } \\
\hline $25-50$ & .140 & .708 & 1.177 & .278 & .667 & .414 & .775 & .379 & .775 & .379 & .775 & .379 \\
\hline \multicolumn{13}{|l|}{$51-65$} \\
\hline \multicolumn{13}{|l|}{ Gender } \\
\hline Male & .208 & .648 & .024 & .877 & .667 & .414 & 3.120 & .077 & 3.120 & .077 & 3.120 & .077 \\
\hline \multicolumn{13}{|l|}{ Female } \\
\hline \multicolumn{13}{|l|}{ Residence } \\
\hline Urban & .037 & .815 & .004 & .950 & .027 & .868 & .531 & .082 & .531 & .082 & .531 & .082 \\
\hline \multicolumn{13}{|l|}{ Rural } \\
\hline \multicolumn{13}{|l|}{ Marital Status } \\
\hline \multicolumn{13}{|l|}{ Married } \\
\hline Single & 5.531 & .063 & 3.257 & .196 & 1.091 & .580 & .048 & .826 & .048 & .826 & .048 & .826 \\
\hline Widow & & & & & & & & & & & & \\
\hline Education & & & & & & & & & & & & \\
\hline Illiterate & .240 & .808 & .957 & $.025 *$ & .000 & 1.000 & .171 & .679 & .171 & .679 & .171 & .679 \\
\hline School education & & & & & & & & & & & & \\
\hline University & & & & & & & & & & & & \\
\hline Occupation & & & & & & & & & & & & \\
\hline Work & .048 & .826 & .112 & .738 & 1.253 & .263 & .048 & .826 & .048 & .826 & .048 & .826 \\
\hline Don't work & & & & & & & & & & & & \\
\hline Income & & & & & & & & & & & & \\
\hline Enough & .171 & 679 & .263 & .608 & .811 & .368 & .208 & .648 & .208 & .648 & .208 & .648 \\
\hline Not enough & & & & & & & & & & & & \\
\hline
\end{tabular}

$* \mathrm{P}<0.05$ (significant) 
Table (8): Relation between total good compliance score and Demographic characteristics for patients with hypertension in both groups throughout the study phases

\begin{tabular}{|c|c|c|c|c|c|c|c|c|c|c|c|c|}
\hline \multirow{5}{*}{$\begin{array}{l}\text { Demographic } \\
\text { characteristics }\end{array}$} & \multicolumn{6}{|c|}{ Study } & \multicolumn{6}{|c|}{ Control } \\
\hline & \multicolumn{2}{|c|}{ Pre } & \multicolumn{2}{|c|}{ Post } & \multicolumn{2}{|c|}{ Follow Up } & \multicolumn{2}{|c|}{ Pre } & \multicolumn{2}{|c|}{ Post } & \multicolumn{2}{|c|}{ Follow Up } \\
\hline & \multicolumn{2}{|c|}{$\begin{array}{c}\text { Total } \\
\text { Compliance }\end{array}$} & \multicolumn{2}{|c|}{$\begin{array}{c}\text { Total } \\
\text { Compliance } \\
\end{array}$} & \multicolumn{2}{|c|}{$\begin{array}{c}\text { Total } \\
\text { Compliance }\end{array}$} & \multicolumn{2}{|c|}{$\begin{array}{c}\text { Total } \\
\text { Compliance }\end{array}$} & \multicolumn{2}{|c|}{$\begin{array}{c}\text { Total } \\
\text { Compliance }\end{array}$} & \multicolumn{2}{|c|}{$\begin{array}{c}\text { Total } \\
\text { Compliance }\end{array}$} \\
\hline & \multicolumn{2}{|c|}{ Good } & \multicolumn{2}{|c|}{ Good } & \multicolumn{2}{|c|}{ Good } & \multicolumn{2}{|c|}{ Good } & \multicolumn{2}{|c|}{ Good } & \multicolumn{2}{|c|}{ Good } \\
\hline & $\mathbf{X}^{2}$ & $\begin{array}{c}\mathbf{P} \\
\text { value }\end{array}$ & $\mathbf{X}^{2}$ & $\begin{array}{c}\mathbf{P} \\
\text { value } \\
\end{array}$ & $\mathbf{X}^{2}$ & $\begin{array}{c}P \\
\text { value } \\
\end{array}$ & $\mathbf{X}^{2}$ & $\begin{array}{c}\mathbf{P} \\
\text { value } \\
\end{array}$ & $\mathbf{X}^{2}$ & $\begin{array}{c}P \\
\text { value } \\
\end{array}$ & $\mathbf{X}^{2}$ & $\begin{array}{c}\mathbf{P} \\
\text { value } \\
\end{array}$ \\
\hline \multicolumn{13}{|l|}{ Age in years } \\
\hline $25-50$ & 1.091 & .580 & 6.790 & $.034 *$ & 4.270 & .118 & 3.210 & .087 & 3.210 & .087 & 3.210 & .087 \\
\hline \multicolumn{13}{|l|}{$51-65$} \\
\hline \multicolumn{13}{|l|}{ Gender } \\
\hline Male & .024 & .877 & 2.667 & .102 & .667 & .414 & .531 & .082 & .531 & .082 & .531 & .082 \\
\hline \multicolumn{13}{|l|}{ Female } \\
\hline \multicolumn{13}{|l|}{ Residence } \\
\hline Urban & 1.430 & .232 & .740 & .857 & .027 & .868 & .710 & .825 & .710 & .825 & .710 & .825 \\
\hline \multicolumn{13}{|l|}{ Rural } \\
\hline Marital Status & 1.177 & .278 & .296 & .586 & .000 & 1.000 & .354 & .535 & .354 & .535 & .354 & .535 \\
\hline \multicolumn{13}{|l|}{ Married } \\
\hline \multicolumn{13}{|l|}{ Single } \\
\hline Widow & & & & & & & & & & & & \\
\hline Education & & & & & & & & & & & & \\
\hline Illiterate & .775 & .379 & .102 & .749 & .667 & .414 & .103 & .780 & .103 & .780 & .103 & .780 \\
\hline School education & & & & & & & & & & & & \\
\hline University & & & & & & & & & & & & \\
\hline Occupation & & & & & & & & & & & & \\
\hline Work & 1.310 & .125 & .440 & .507 & 3.683 & .055 & 1.430 & .232 & 1.430 & .232 & 1.430 & .232 \\
\hline Don't work & & & & & & & & & & & & \\
\hline Income & & & & & & & & & & & & \\
\hline Enough & 3.120 & .077 & .867 & $.035 *$ & .360 & .548 & .024 & .877 & .024 & .877 & .024 & .877 \\
\hline Not enough & & & & & & & & & & & & \\
\hline
\end{tabular}

$* \mathrm{P}<0.05$ (significant) 
Table (9): Relation between total good compliance score and HBM for patients in both groups throughout the study phases

\begin{tabular}{|c|c|c|c|c|c|c|c|c|c|c|c|c|}
\hline \multirow{5}{*}{ HBM } & \multicolumn{6}{|c|}{ Study } & \multicolumn{6}{|c|}{ Control } \\
\hline & \multicolumn{2}{|c|}{ Pre } & \multicolumn{2}{|c|}{ Post } & \multicolumn{2}{|c|}{ Follow Up } & \multicolumn{2}{|c|}{ Pre } & \multicolumn{2}{|c|}{ Post } & \multicolumn{2}{|c|}{ Follow Up } \\
\hline & \multicolumn{2}{|c|}{$\begin{array}{c}\text { Total } \\
\text { Compliance }\end{array}$} & \multicolumn{2}{|c|}{$\begin{array}{c}\text { Total } \\
\text { Compliance }\end{array}$} & \multicolumn{2}{|c|}{$\begin{array}{c}\text { Total } \\
\text { Compliance }\end{array}$} & \multicolumn{2}{|c|}{$\begin{array}{c}\text { Total } \\
\text { Compliance }\end{array}$} & \multicolumn{2}{|c|}{$\begin{array}{c}\text { Total } \\
\text { Compliance }\end{array}$} & \multicolumn{2}{|c|}{$\begin{array}{c}\text { Total } \\
\text { Complianc }\end{array}$} \\
\hline & \multicolumn{2}{|c|}{ Good } & \multicolumn{2}{|c|}{ Good } & \multicolumn{2}{|c|}{ Good } & \multicolumn{2}{|c|}{ Good } & \multicolumn{2}{|c|}{ Good } & \multicolumn{2}{|c|}{ Good } \\
\hline & $X^{2}$ & $\begin{array}{c}\mathbf{P} \\
\text { value }\end{array}$ & $\mathbf{X}^{2}$ & $\begin{array}{c}\mathbf{P} \\
\text { value }\end{array}$ & $\mathbf{X}^{2}$ & $\begin{array}{c}\mathbf{P} \\
\text { value }\end{array}$ & $\mathbf{X}^{2}$ & $\begin{array}{c}\mathbf{P} \\
\text { value }\end{array}$ & $\mathbf{X}^{2}$ & $\begin{array}{c}\mathbf{P} \\
\text { value }\end{array}$ & $\mathbf{X}^{2}$ & $\begin{array}{c}\mathbf{P} \\
\text { valu }\end{array}$ \\
\hline \multicolumn{13}{|l|}{ Perceived } \\
\hline Severity & 1.92 & .909 & 2.15 & $.025 *$ & 1.65 & .583 & 1.87 & .640 & 1.22 & .520 & 1.32 & .612 \\
\hline \multicolumn{13}{|l|}{ Perceived } \\
\hline susceptibility & .394 & .778 & .452 & .683 & .574 & .153 & 2.23 & .760 & 3.88 & .570 & 3.88 & .570 \\
\hline \multicolumn{13}{|l|}{ Perceived } \\
\hline Benefits & 3.03 & .952 & 3.27 & $.036 *$ & 2.45 & 1.13 & .207 & .884 & .076 & .487 & .057 & .466 \\
\hline Perceived & 2.39 & .763 & 1.39 & .952 & .782 & .202 & 2.23 & .352 & 2.44 & .644 & 2.44 & .644 \\
\hline \multicolumn{13}{|l|}{ Barriers } \\
\hline Self-Efficacy & 2.14 & .848 & 3.59 & $.012 *$ & 1.74 & .765 & 2.17 & .382 & 2.17 & .382 & 2.17 & .382 \\
\hline $\begin{array}{l}\text { Cues to } \\
\text { Action }\end{array}$ & 2.38 & .747 & 1.75 & .458 & .832 & .222 & 2.84 & .846 & 2.84 & .846 & 2.84 & .846 \\
\hline
\end{tabular}

$* \mathrm{P}<0.05$ (significant)

Table (10): Correlation coefficient between knowledge, practice and compliance of study group throughout the study phases $(n=40)$

\begin{tabular}{|l|c|c|c|c|c|c|}
\hline \multirow{2}{*}{\multicolumn{1}{|c|}{ Item }} & \multicolumn{2}{c|}{ Knowledge } & \multicolumn{2}{c|}{ Practice } & \multicolumn{2}{c|}{ Compliance } \\
\cline { 2 - 7 } & $\mathbf{r}$ & $\mathbf{p}$ & $\mathbf{r}$ & $\mathbf{p}$ & $\mathbf{r}$ & $\mathbf{p}$ \\
\hline Pre knowledge & - & - & .217 & .179 & .257 & .170 \\
\hline Pre practice & .217 & .179 & - & - & .861 & .369 \\
\hline Pre compliance & .257 & .170 & .861 & .369 & - & - \\
\hline Post knowledge & - & - & .473 & $.002^{* *}$ & .344 & $.030^{*}$ \\
\hline Post practice & .473 & $.002^{* *}$ & - & - & .668 & $.001^{*}$ \\
\hline Post compliance & .344 & $.030^{*}$ & .668 & $.001^{*}$ & - & - \\
\hline FU knowledge & - & - & .319 & $.045^{*}$ & .292 & .068 \\
\hline FU practice & .319 & $.045^{*}$ & - & - & .223 & .236 \\
\hline FU compliance & .292 & .068 & .223 & .236 & - & - \\
\hline
\end{tabular}

*r: Spearman correlation coefficient

Interpretation of $* \mathbf{r}$ :
Weak $(0.1-0.24)$
Intermediate $(0.25-0.74)$
Strong $(0.75-0.99)$ 


\section{DISCUSSION}

Hypertension is often an asymptomatic disorder characterized by persistent elevation of the SBP at a level of $140 \mathrm{mmHg}$ or higher and DBP at a level of $90 \mathrm{mmHg}$ or higher [16]. High BP is a major risk factor for heart disease, congestive heart failure, stroke, impaired vision, and kidney disease. Clinical manifestations will become apparent, and patients will eventually complaint about persistent headaches, fatigue, dizziness, palpitations, flushing, blurred or double vision, or epistaxis [17].

Health Belief Model (HBM) is widely used intervention programs aiming to change behaviors, especially those related to dietary practices, infectious disease, smoking cessation and high blood pressure screening. The model assumes that a person's beliefs about health are determinants of the possibility of an individual to make changes in the lifestyle behaviors. Therefore, health education utilizing HBM is a crucial factor in preventing illness, provision of information will help them in taking care of themselves and their family [18].

Discussion of the results will cover these areas in the following sequence; demographic characteristics and medical history of adult patients with hypertension under the study, patients' behaviour toward life style habites, patients' knowledge about hypertension, compliance of patients with hypertension toward therapeutic regimen, patients' practice regarding Blood pressure measurement, relaxation exercises, Factors affecting patient's compliance based on Health Belief model and relation and correlation between different variables.

Demographic characteristics and medical history for patients with hypertension in both study and control groups including; gender, age, residence, marital Status, education, occupation and income were matched and this help to control other variables that may affect the outcome of the study[6]. The present study clarified that none the patients in both study and control groups had satisfactory level of knowledge regarding hypertension, in preprogram phase. This finding was agree with Elaskary [19] who reported that there was a significant increase in knowledge level post program and in follow up after three months compared to preprogram. This could be explained by the fact that patients didn't receive enough information from health care providers or/ and the health professionals didn't find the time to provide them with enough information and also the majority of the studied patients were illiterate. After implementation of the health promotion program, the results of the study showed that there was a statistical significant increase in patients' level of knowledge regarding hypertension, in study group. This finding agrees with Al-Wehedy Abd Elhameed and Abd ElHameed [20] who illustrated that lifestyle modification sessions improved the knowledge scores of the study group of hypertensive patients with highly statistical significant difference between study and control. Ambaw, Alemie, Yohannes and Mengesha [21] concluded that right knowledge about hypertension and its treatment creates a clear understanding and avoids confusion about the treatment and the disease condition. Knowledge about hypertension and its treatment was found to be positively associated with adherence behavior. Patients with better awareness were more likely to adhere to their treatment.

In this study there was marked improvement in compliance score related to hypertension after the educational program. This approach is also supported by Ahmed [22] who reported that patient education plays a fundamental role in successful management of hypertension. Consequently, the failure to establish an effective communication with patients is associated with non-adherence and poor BP control. These mean that the provision of more detailed information about hypertension was associated with better compliance to treatment and BP control.

It was observed that patients had unsatisfactory level of practice about Blood pressure measurement, relaxation exercises preprogram, which significantly improved after patients' involvement in the education sessions. This finding was in the same line with Ez Elregal [23] in Ain Shams University who reported in a study about "Promoting Health behaviors of Clients with Hypertensive Kidney Disease by Using Health Promotion Model" that there was improvement of practice about Blood pressure measurement, breathing exercises and Progressive Muscle Relaxation after program. It might be due to that the patient required enough instructions about practices that help in minimizing occurrence of stress.

The Health Belief Model, which is widely used to study health behaviour, formed the theoretical framework for this study. The core components of the Model are perceived susceptibility, perceived severity, perceived benefits and perceived barriers. The Model postulates that health behaviour 
towards a disease or treatment is succinctly influenced by the extent to which individuals believe they are susceptible to the disease and how severe they believe the disease is, the benefits they stand to gain by adopting the required health behaviour and the barriers standing in the way of adopting the required health behaviour. The expanded version of the Model also includes variables such as self-efficacy and cues to action [24]. The finding of the present study was expected in relation to HBM model constructs, that there were statistical differences for all constructs except for cues to action post intervention among the study group compared with the control group. Similar finding was reported by Elaskary [19] who reported that positive changes in the beliefs of patients in the intervention group in comparison to the control group for all HBM constructs except for cues to action.

The current study illustrated that there was statistical significant relation between total knowledge score and educational level in study group in post program phase. This finding is in contrast with Ikea, Aniebueb and Aniebuec [25] who showed that there was no statistical difference regarding knowledge score and educational level. The result of the study showed that there was statistical significant relation between total knowledge score and duration of disease in study group. Those individuals who had more years of illness showed a greater improvement in knowledge with their medical regimen after completion of the educational program. This could be explained as, with increasing duration of illness, the patients recognize that their disease is lifelong one and not curable. For these reasons, the patients' knowledge improved with duration of disease. This finding agrees with EDO [26] who found that there was a statistical significant relation between knowledge and duration of disease.

The result of the current study indicated that there was statistical significant relation between the patients' compliance and their age in study group in post program phase. This finding is in the same line with Ahmed [21] who reported that age of patients was a significantly correlated with compliance rate. These may be explained by the fact that older patients tend to be more scared of disease; consequently, they have fear of death than younger ones, so they comply with the medical regimen imposed by the disease. Also this contradiction may be explained as these studies included different age categories younger and older age. In addition, younger participants were more non-adherent to their treatment regimen possibly due to ignorance of the true nature of hypertension or denial of the existence of the disease.

According to relation between the patients' compliance and their income the current results showed that, there was statistical significant relation between the patients' compliance and their income in study group in post program phase. In investigator opinion this may be due to the patients with insufficient income can't adhere with therapeutic regimen because they don't have the money and cannot afford the cost of medications and transport costs to health centers. They often have to barter the pressing need to provide food for the family rather than procure medications. This finding was in accordance with Abd Allah [27] who found that financial support for patients improved their compliance.

According to relation between patients' compliance and HBM, the current results showed that, there was statistical significant relation between patients' compliance and perceived severity in study group in post phase. This finding matched with Mahrous [28] who also found that patients complying with their treatment depend on the degree to which they perceive themselves to be susceptible to the disease or its complications and their perceptions of the severity of the condition. This could be attributed to the persons who perceive hypertension to be a serious disease would be more compliant with medication and lifestyle modifications than those who do not hold this perception.

Concerning to relation between patients' compliance and HBM, the present study revealed that, there was statistical significant relation between patients' compliance and Self-Efficacy in study group in post phase. This finding was in accordance with $\mathrm{Hu}, \mathrm{Li} \&$ Arao [29] who found self-efficacy has been recognized as a major predictor of self-care behavior for chronic disease management as adopting healthy diet and regular exercise, reported better health status, and lower psychological distress. This may be due to the fact that most hypertension patients already know what actions they should take, such as weight loss, smoking cessation or participating in exercise activities, but knowledge is insufficient to stimulate actions. Patients need to believe in their capability and have confidence to perform the expected behaviour.

As regard to the relation between patients' knowledge and compliance, the present study revealed that there was positive correlation 
between total knowledge \& total compliance for patients in study group in post phase. This finding is supported by Al-Jbour, Abu Kamel \& Barhoom [30] who stated that increasing patients' knowledge about the disease can achieve the goal of treatment, empower patients to make decision about their treatment, and can empower their motivation and intention to compliance with the treatment regimen. Also finding go in line with Duncan, Howe L, Manakusa \& Purdy [31] who also found that Providing information about the disease and treatment regimen appears to be sufficient for compliance, and non-compliance is often attributed to inadequate knowledge.

While this finding was in contrast with Hassan [32], Ahmed [33] who reported that there was no relation between knowledge about HTN and compliance with therapeutic treatment to control BP. This is may be justified by knowledge was not enough to achieve compliance and changing in lifestyle because knowledge is not the only component to achieve the goal, but also positive attitude and behaviors.

On summary, the results of this study support the hypothesis that the knowledge, compliance and practice scores of patients who received health beliefs model were higher than that of a control group. There was a significant improvement in knowledge, compliance and practice of patients with hypertension post and follow up phase of program in study group.

Funding: None.

Conflicts of interest: None.

Ethical approval:Approved .

\section{REFERENCES}

1- Adebayo1 A, Rotkangmwa $\mathrm{O}$ and Shalkur D: Hypertension related practices and compliance to anti-hypertensive therapy among hypertensive patients in tertiary health care facilities in Jos, North-Central Nigeria, World Journal of Pharmaceutical Sciences, 2015, 3(6): 1151-1158.

2- Eshah N and Al-daken L: Assessing Publics' Knowledge about Hypertension in a CommunityDwelling Sample. Journal of Cardiovascular Nursing; 2015, 33 (4): 1-8.

3- World Health Organization: A global brief on hypertention Available at: http://www.who.int/ cardiovascular_diseases/publications/global_brief_hypert ensi on/en. Accessed on 16/3/2016 at 3:30 pm, 2015.

4- Khosravizade A Hassanzadeh A and Mostafavi F: The Impact of Self-Efficacy Education on Self-
Care Behaviors of Low Salt and Weight Setting Diets in Hypertensive Women Covered by HealthCare Centers of Dehaghan in 2013. J Pak Med Assoc; 2015, 65 (5): 506- 511.

5- Ibrahim M: Problem of Hypertension in Egypt, The Egyptian Heart Journal, 2013, 65(3) PP 233-234, Available at:http://www.egyptheartjournal.com/ article, Accessed on: January, 6/2015.

6- Awad E, Gwaied B, Fouda L, and Essa H: Compliance of Hypertensive Patients with Treatment Regimen and Its Effect on Their Quality Of Life. IOSR Journal of Nursing and Health Science; 2015, 4(2): 26-36.

7- Mersal F and Mersal N: Effect of Evidence Based Lifestyle Guidelines on Self Efficacy of Patients with Hypertension, Int. J. Curr. Microbiol. App. Sci, 2015, 4(3)Pp 244-263.

8- Alton, Mat, Waiti, Jordan, Signal, Louise, Thomson and George: Identifying barriers to promoting healthy nutrition in New Zealand primary schools. Health Education Journal, 2015, 69 (1): 84-94.

9- Khoramabadi M, Dolatian M and Hajian S: Effects of Education Based on Health Belief Model on Dietary Behaviors of Iranian Pregnant Women. Global Journal of Health Science; 2016, 8 (2): 230-239. ISSN 1916-9736 E-ISSN 1916-9744.

10- American Heart Association: Heart disease and stroke statistics: 2004 update, Available at: http:// www.americanheart.org. Accessed on 12/6/2016 at $3 \mathrm{pm}, 2004$.

11- Lynn P and LeBon M: Skill Checklists for Taylor's Clinical Nursing Skills :A Nursing Process Approach, 3rd ed, Wolters Kluwer Health Lippincott Williams \& Wilkins, United States of America, 2011, P 11. P 120.

12- Sue C, DeLaune and Ladner P: Fundamentals of Nursing: Standards and Practice, Fourth Edition, Delmar Cengage Learning, United States of America, 2011, PP 833-852.

13- Dave A and Makwana J: The Effect of Progressive Muscular Relaxation and Psychological Counseling on Physiological Parameters during Surgical Stress. Journal of Evidence based Medicine and Healthcare; 2015, 2(43): 7709-7716.

14- Madhava $R$ and Deepa $H$ : An Impact of Jacobson's Progressive Muscle Relaxation (JPMR) in Managing the Perceived Stress Level among College Students. International Journal of Research in Medical \& Applied Sciences; 2015, 1 (2): 2454-3667.

15- Burns $\mathrm{N}$ and Grove $\mathrm{S}$ : The practice of nursing research: conduct, critique \& utilisation; 5th edition. Philadelphia: W.B.Saunders, 2005. 
16- Ignatatvicius D and Workman M: Medical-Surgical Nursing Patient Centre Collabrative Care, Hypertension, 7th ed.; 2014, Pp.777-85. Elsevier, Philadelphia.

17- Kazuaki S, Katsuyuki A, Toshiro F, Naoyuki H, Jitsuo $\mathrm{H}$ and Masatsugu $\mathrm{H}$ : The Japanese Society of Hypertension Guidelines for the Management of Hypertension (JSH 2014). Hypertension Research; 2014, 37: 253-392.

18- Kazemi A, Ehsanpour S, Zahraei N, Hasanzadeh A, Beigi and Malverdi M: Impact of health belief modification on intention to make smoke free home among pregnant women. J Res Med Sci. 2011 Jun; 16(6): 724-732.

19- Elaskary E: Impact of Health Promotion program on compliance with therapeutic regimen among hypertensive patients in Gaza Strip, Unpublished Doctoral Thesis. Faculty of Nursing, Cairo University, 2011.

20- Al-Wehedy A, Abd Elhameed S, and Abd ElHameed D: Effect of Lifestyle Intervention Program on ControllingHypertension among Older Adults, Journal of Education and Practice, 2014, 5 (5), Pp 61-71.

21- Ambaw A, Alemie A, Yohannes M and Mengesha B: Adherence to antihypertensive treatment and associated factors among patients on follow up at University of Gondar Hospital, Northwest Ethiopia. BMC Public Health, 2012, 12: 282.

22- Ahmed B: Effect of Educational Program on Knowledge, Self-Efficacy and Compliance of Patients with Essential Hypertension toward Therapeutic Regimen, UnPublished doctorate Thesis, Faculty of Nursing, Port Said University, 2017.

23- Ez Elregal F: Promoting Health behaviors of Clients with Hypertensive Kidney Disease By Using Health Promotion Model. Unpublished Doctoral Thesis. Faculty of Nursing, Ain Shams University, 2013.

24- Coltrell R and Mckenzie J: Health promotion and education research methods. $2^{\text {nd }}$ edition. Ones \&Bartlett, Boston \& London, 2011, P67.
25- Ikea S Aniebueb P and Aniebuec U: Knowledge, Perceptions and Practices of Lifestyle-Modification Measures among Adult Hypertensive in Nigeria. Transact. Royal Soc. Trop. Med. Hyg; 2010, 104(9): 55-60.

26- EDO T: Factors affecting compliance with antihypertensive drug treatment and required lifestyle modifications among hypertensive patients on Praslin Island, Published thesis, Degree Master of Public Health, University of South Africa. Available at: www.elsevier.com/locate/socscimed. Accessed on $7 / 6 / 2016$ at 7 am, 2009.

27- Abd Allah D: Factors Affecting Compliance for Haemodialysis Patients Toward Therapeutic Regimen, Unpublished Master Thesis. Faculty of Nursing, Zagazig University, 2013.

28- Mahrous F: Factors Affecting Compliance of Hypertensive Patients toward Therapeutic Regimen, Life Science Journal, 2015, 12(10) Pp62-69.

29- $\mathrm{Hu} \mathrm{H}, \mathrm{Li} \mathrm{G}$ and Arao T: Validation of a Chinese Version of the Self-Efficacy for Managing Chronic Disease 6-Item Scale in Patients with Hypertension in Primary Care. ISRN Public Health; Article ID 298986, 6 pages. Available at: http://www. patient.info/doctor/Jugular-Venous-Pressure.htm. Accessed on 19/5/2016 at 2 pm, 2013.

30- Al-Jbour B, Abu Kamel A and Barhoom H: Knowledge about Hypertension and Antihypertensive Medication Compliance in a Jordanian Community Sample. Journal of Education and Practice; 2013, 4 (24):81-87.

31- Duncan P, Howe L, Manakusa Z and Purdy S: Determinants of Blood Pressure Control in Rural KwaZulu-Natal, South Africa. South African Family Practice; 2015, 56(6): 297-304.

32- Hassan N: Compliance of Hypertensive Elderly Patients with Treatment Regimen. Unpublished Master Thesis. Faculty of Nursing, Alexandria University, 2009.

33- Ahmed B: Factors Affecting Compliance of Patients with Essential Hypertension toward Therapeutic Regimen. UnPublished thesis, Master degree Thesis, Faculty of Nursing, Port Said University, 2014. 